\title{
Robust Control for the Vibration Attenuation of Superstructures
}

\author{
Wen-Jeng Hsueh*, Member
}

\begin{abstract}
Summary
A robust control methodology applied for active vibration reduction on superstructures using the $\mathrm{H}_{\infty}$ synthesis is presented. Based on this technique, the control law may be calculated according to the required objective. The designed control system will automatically meet the performance and robustness properties, which are given in the specifications. Finally, the superstructure of a cargo ship subject to wind gust and foundation oscillation is investigated. The results show that the active vibration control system designed by this scheme has an effective vibration attenuation for both excitations. In addition, this controller will maintain high performance even when there is a large perturbation in the system parameter.
\end{abstract}

\section{Introduction}

Ship vibration has been an interesting subject to engineers, builders and owners more than a century. Particularly, vibration at the superstructure has attracted more attention because of its immediate effect on the comfort of passengers and crew. Since the superstructure is directly attach on the hull, the hull vibration becomes a dominant excitation source. Unfortunately, this excitation source has become more severe recently because the high screw propeller and long stroke diesel engine have become more widely used ${ }^{1}$.

In order to eliminate the vibration of the superstructures, several methods have been developed by pioneers in the field. One of the major methods is to decrease the intensity of the excitation sources, such as using engine isolators or absorbers to reduce the engine generating force and moment ${ }^{2)}$. The other way is to design the passive type neutralizer ${ }^{3), 4}$ or the active type absorber ${ }^{5), 6)}$ to take the vibration energy away from the hull. Then the excitation transferred from the hull will be eliminated. This method can offer a good operation environment for the crew as well as lower failure rates in the structures. However, the complex configuration and high power capacity required for the actuator system is its primary disadvantage and the efficiency of this method is very limited for reducing the fore-aft

* Department of Naval Architecture and Ocean Engineering, National Taiwan University, Taiwan, ROC.

Received 10th July 1997

Read at the Autumn meeting 14, 15th Nov. 1997 vibration of the superstructure, which is one of the most dominant vibration types.

For these reasons, several devices have been developed and installed on the superstructure to directly attenuate the motion of the superstructure, including the classical passive type, the adjustable passive type $^{7), 8), 9)}$ and the active type absorbers ${ }^{10), 11), 12}$. Of these devices, the active type absorber has become more important because it can offer higher efficiency in vibration reduction. When the active absorber system is selected, the vibration of the superstructures can be attenuated by a suitable active force, which is calculated by the controller according to the behavior of the structure. Thus the efficiency of the active system is dependent on the control law of the controller. In most of the previous applications, the LQR method are the most widely used on the active absorber for superstructures. By the LQR method, the control law is calculated by the minimization of a performance function, which is the function of the weighting matrices with respect to the motion of the superstructure and the movement of the actuator. However, the determination of the weighting matrices according to the required performance is difficult work. Recently, an $\mathrm{H}_{\infty}$ synthesis based on a singular value theorem has been proposed for the automatic control engineering ${ }^{13), 14), 15)}$. Through this scheme, the control law can be calculated based on the sensitivity and robustness functions, which are assigned directly according to the required specification of the dynamic system. Thus the designer can easily obtain a suitable control law to meet the requirements of the disturbance rejection ability and the robustness property based on this scheme. In this paper the $\mathrm{H}_{\infty}$ synthesis is used to design the control law for the active 
vibration reduction of the superstructure. In order to understand the efficiency of this methodology, the excitation of the wind gust and base oscillation is considered in the numerical simulation. In addition, the parameter variation of the system is also analyzed to understand the robustness property of this scheme.

\section{Theory}

\subsection{Structure Dynamics}

The structure model for the vibration analysis of the superstructure of ships is modeled as a one degree of freedom dynamic system shown in Fig. 1 . Let $m_{s}$ be the generalized mass of the superstructure, which is attached to the main hull by a linear spring and damper with constant stiffness and damping coefficients $k_{s}$ and $b_{s}$. The external excitations including wind gust and support oscillation, caused by motion of the hull, are considered. If a pair of tendons with actuators are connected between the top of the superstructure and the hull ${ }^{10), 16)}$, an active force $f_{a}(t)$ generated by the actuators is acting on the superstructure. The equation of the motion for the superstructure is given by

$$
m_{s} \frac{d^{2} y_{s}(t)}{d t^{2}}+b_{s} \frac{d \tilde{y}_{s}(t)}{d t}+k_{s} \tilde{y}_{s}(t)=f_{w}(t)+f_{a}(t)
$$

where $\tilde{y}_{s}(t)$ is the relative generalized displacement of the superstructure with respect to the supported hulls. $f_{w}(t)$ is the generalized excitation force of the wind gust. In general, the active force generated by the actuator has a time lag response after the command. Thus, the action of the actuator can be represented by a first order differential equation such as

$$
f_{a}(t)+\tau \frac{d f_{a}(t)}{d x}=k_{d} v_{d}(t)
$$

where $v_{d}(t)$ is the input command signal for the controller and $\tau$ and $k_{d}$ are the lag time constant and gain constant of the actuator. Taking the Laplace transformation of the Eqs. ( 1 ) and (2), the frequency domain of the dynamic system may be written

$$
\begin{aligned}
& Y_{s}(s)=G_{s}(s) F_{a}(s)+D(s) \\
& D(s)=G_{s}(s) F_{w}(s)+G_{h}(s) Y_{h}(s)
\end{aligned}
$$

where $s$ is a complex variable. $Y_{s}(s), Y_{h}(s), D(s), F_{w}(s)$ and $F_{a}(s)$ are the Laplace transformation of $y_{s}(t), y_{h}(t)$, $d(t), f_{w}(t)$ and $f_{a}(t) . y_{h}(t)$ is the absolute displacement

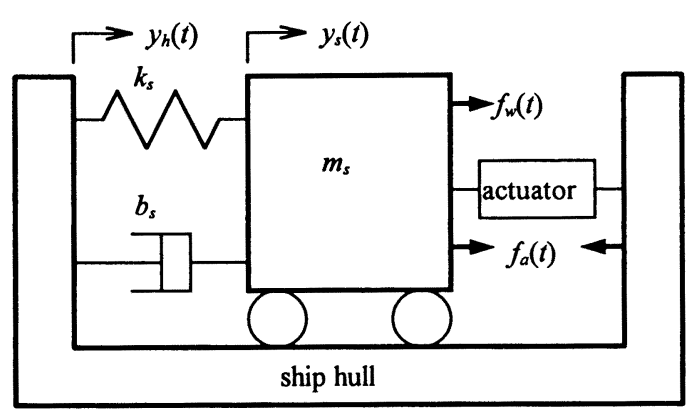

Fig. 1 Dynamic model of the superstructure of the foundation of the superstructure. $d(t)$ is the total excitations caused by wind gust and support oscillation. $G_{s}(s)$ and $G_{h}(s)$ are the transfer function of the superstructure displacement with respect to the wind gust load and excitation due to the hull vibration given by

$$
\begin{aligned}
G_{s}(s) & =\frac{k_{a}}{m_{s}(s+1 / \tau)\left(s^{2}+2 \zeta_{s} \omega_{s} s+\omega_{s}^{2}\right)} \\
G_{h}(s) & =\frac{\left(2 \zeta_{s} \omega_{s} s+\omega_{s}^{2}\right)}{\left(s^{2}+2 \zeta_{s} \omega_{s} s+\omega_{s}^{2}\right)}
\end{aligned}
$$

where $\zeta_{s}$ and $\omega_{s}$ are the generalized damping ratio and natural frequency of the superstructure defined by

$$
\begin{aligned}
& \omega_{s}=\sqrt{\frac{k_{s}}{m_{s}}} \\
& \zeta_{s}=\frac{b_{s}}{2 \sqrt{m_{s} k_{s}}}
\end{aligned}
$$

\section{2 Controller Design}

The objective of this problem is to find a control law, which is used to generate a suitable active force to attenuate the vibration approaching the required level. Assume a feedback control loop is considered for the dynamic system shown in Fig. 2. The transfer function from disturbance input $D(s)$ to the output $Y_{s}(s)$ is just equal to the transfer function from input command $R(s)$ to the error $E(s)$, called the sensitivity function matrix, $S(s)$, defined by

$$
S(s)=\left(I+F_{c}(s) G_{s}(s)\right)^{-1}
$$

So the singular value of the sensitivity function may be used to determine the performance of the disturbance attenuation. From the singular value theory, the relationships between the disturbance attenuation and the sensitivity function are given by

$$
\bar{\sigma}(S(j \omega)) \leq\left|W_{1}^{-1}(j \omega)\right|
$$

where $\bar{\sigma}$ is the greatest singular value, $\left|W_{1}^{-1}(j \omega)\right|$ is the disturbance attenuation factor. In addition, the complementary value of the sensitivity function is just equal to the transfer function from input command $R(s)$ to the output $Y_{s}(s)$, called the complementary sensitivity, $C(s)$, expressed by

$$
C(s)=F_{c}(s) G_{s}(s)\left(I+F_{c}(s) G_{s}(s)\right)^{-1}
$$

This complementary sensitivity function can be used to determine the stability margin for the multiplicative uncertainty of the system. From the robust theorem,

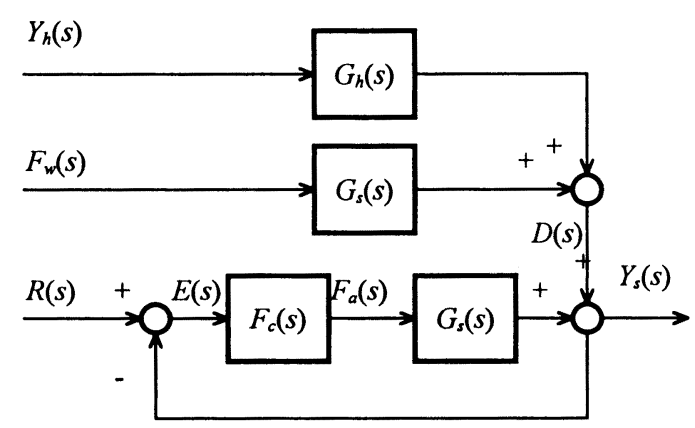

Fig. 2 Flow chart of the structure with acitve control system 
the relationships between the stability margin and the complementary sensitivity function is given by

$$
\bar{\sigma}(C(j \omega)) \leq\left|W_{2}^{-1}(j \omega)\right|
$$

where $\left|W_{2}^{-1}(j \omega)\right|$ is the sizes of the largest anticipated perturbation of the system. Equations ( 8 ) and (10) can be combined into a single form by the infinity norm specification

$$
\left\|\left[\begin{array}{l}
W_{1} S \\
W_{2} C
\end{array}\right]\right\|_{\infty} \leq 1
$$

where \|\|$_{\infty}$ is the least upper bound of the frequency dependent singular values, called the $H_{\infty}$ norm. If the error and the output of the closed loop system are multiplied by the functions of $W_{1}$ and $W_{2}$, the block diagram of the system can be rearranged into a special form including the augmented plant $P(s)$ and controller $F_{c}(s)$ as shown in Fig. 3. Thus the condition of Eq. (11) can be expressed by the constraint of the closed loop transfer function of the new system

$$
\mid T_{Y_{1} U_{1}} \|_{\infty} \leq 1
$$

where $T_{Y_{1} U_{1}}$ is the transfer function from the input $U_{1}$ to the output $Y_{1}$. Thus, determining the controller which can achieve results under the constraint condition of Eqs. ( 8 ) and (10) is the same as determining the controller which satisfies the condition of Eq. (12). Under this new equation and block diagram, the vibration reduction problem becomes a typical $\mathrm{H}_{\infty}$ small gain problem, which can be solved by an $\mathrm{H}_{\infty}$ optimal control synthesis.

\section{Simulation and Results}

In order to understand the performance of the active vibration control system utilizing the robust controller designed in this paper, a superstructure of a cargo ship is considered for numerical analysis. For simplification, only the dominant fore-aft vibration mode is considered. With respect to this mode, the natural frequency is $3.0 \mathrm{~Hz}$ and the damping ratio is $1 \%$. The generalized mass with respect to this vibration mode is 200 ton. The gain constant and the lag time constant of the actuator

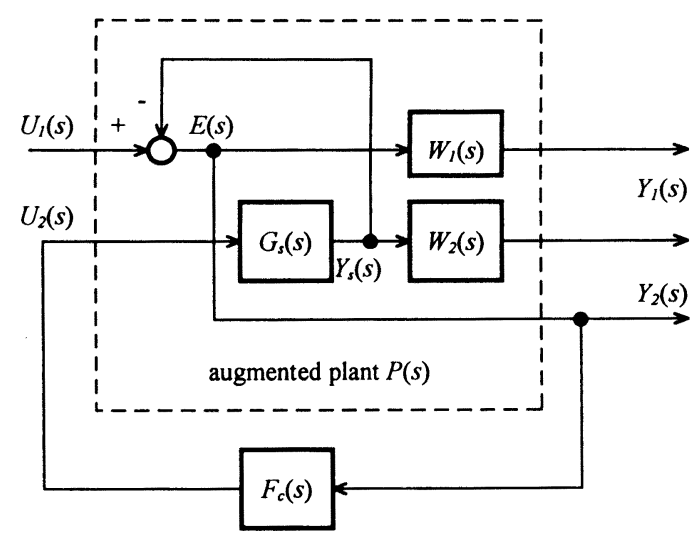

Fig. 3 Block diagram for $\mathrm{H}_{\infty}$ formulation of the dynamic system are $7.1 \mathrm{ton} / \mathrm{mV}$ and $0.02 \mathrm{sec}$. The $\mathrm{H}_{\infty}$ method is used to design the controller for the vibration attenuation of the superstructure. The design objective is to achieve at least 10:1 reduction in position error with respect to the open loop performance in the presence of disturbances over the frequency range of 0 to $5 \mathrm{~Hz}$. Moreover, this control system requires a $20 \mathrm{~dB}$ per decade roll-off above $50 \mathrm{~Hz}$ to achieve the required robustness. To form the augmented plant for analysis, the weighting functions $W_{1}(s)$ and $W_{2}(s)$ should be assigned firstly to meet the required specification. According to the specification described above, the weighting functions $W_{1}=0.1(s+300)^{2}(s+30)^{-2}, \quad W_{2}=10(s+100)(s+3000)^{-1}$ are selected. Then, the control law can be obtained by the $\mathrm{H}_{\infty}$ synthesis.

The wind gust is first considered as the excitation source in these numerical studies. Fig. 4 is the frequency response of the superstructure caused by generalized wind load. The peak value of the displacement is reduced from $7 \mathrm{~mm} /$ ton to $0.6 \mathrm{~mm} /$ ton by the active system. The maximum value of the active force generated by the actuator is less than 1.1 ton/ton. When the support oscillation is considered as the excitation, Fig. 5 represent the peak response of the original structure is $50 \mathrm{~mm} / \mathrm{mm}$ which will be decreased more than by $90 \%$ by the active system. In this case, the required active force is less than $8 \mathrm{ton} / \mathrm{mm}$. Fig. 6 is the time domain response based on the sinusoidal wind gust load, which is the combination of three 1 ton amplitude with different frequency, $1.1 \mathrm{~Hz}, 3 \mathrm{~Hz}$ and $5.4 \mathrm{~Hz}$. We see that the acceleration of the superstructure will be magnified to $0.25 \mathrm{~g}$ amplitude in the original structure. This high acceleration response may be reduced to $0.01 \mathrm{~g}$ if the active system is used. The maximum value of the active force required is nearly 3 ton. In the case of

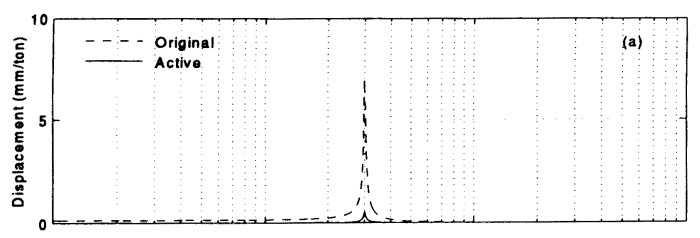

(a) displacement

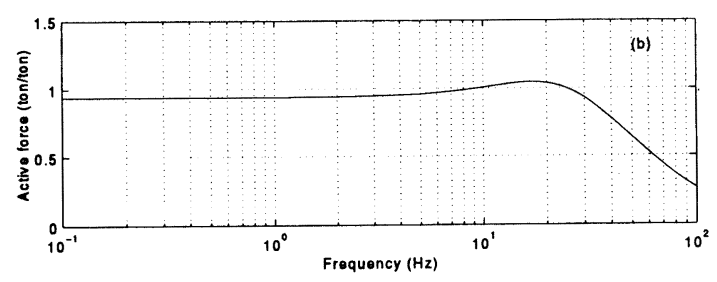

(b) active force

Fig. 4 Transfer function of the structure subject to wind gust ( a ) displacement ( $b$ ) active force 


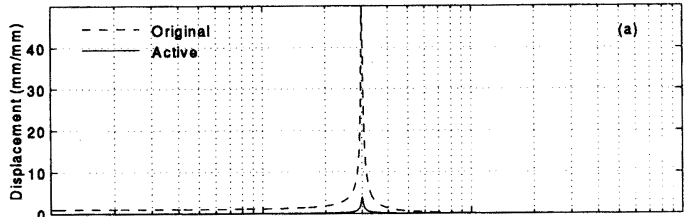

( a ) displacement

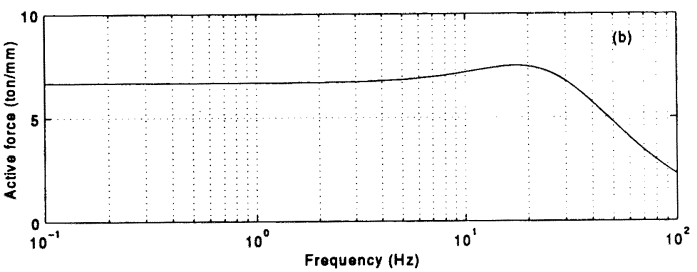

(b) active force

Fig. 5 Transfer function of the structure subject to support oscillation (a) displacement (b) active force

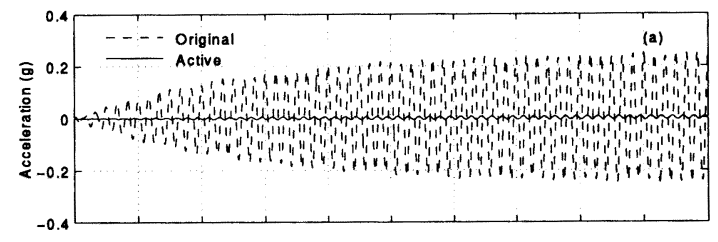

(a) acceleration

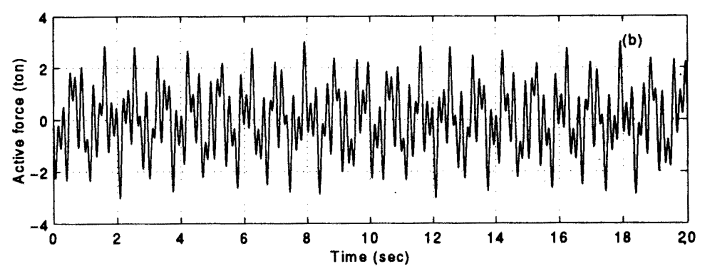

(b) active force

Fig. 6 Time response by a sinusoidal wind gust (a) acceleration ( $b$ ) active force

hull induced vibration, three combined motion of hull, which has $1 \mathrm{~mm}$ amplitude and the same frequencies as the wind load studied in previous case is considered. Fig. 7 shows that high acceleration will be induced if no vibration attenuation system have been installed. However, this high value acceleration can be reduced $90 \%$ by the active system with the designed controller. The amplitude of the active force generated is near 20 ton. From Fig. 4 to 7 , we see that the vibration of the superstructure caused by support oscillation may be more dominant than the wind load. Since the robust characteristic is a very important property of the $\mathrm{H}_{\infty}$ controller, the results under some parameter variation

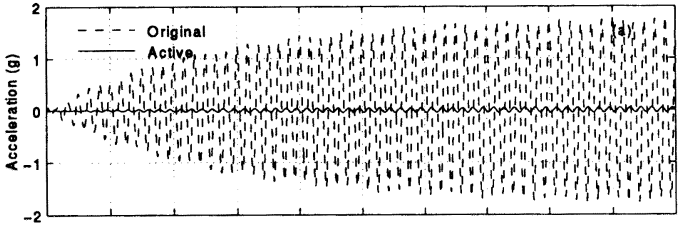

(a) acceleration

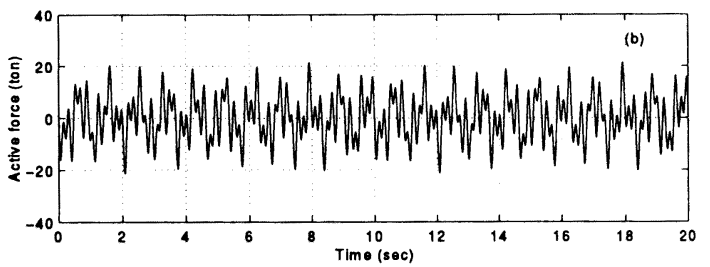

(b) active force

Fig. 7 Time response by a sinusoidal support oscillation ( a ) acceleration ( $b$ ) active force

are considered in the following simulations. If the lag time constant of the actuator is changed from $0.02 \mathrm{sec}$ to $0.1 \mathrm{sec}$, Figs. 8 shows that the responses of the system with a changed time constant for the excitations of wind gust and support oscillation are nearly the same as those without a changed parameter. Fig. 9 is the response for the excitations of wind gust and support oscillation if the natural frequency of the superstructure used for the controller design has a $30 \%$ error or less with respect to that of the true structure. From the results, we find that this controller perform well in term of the vibration attenuation of the structure.

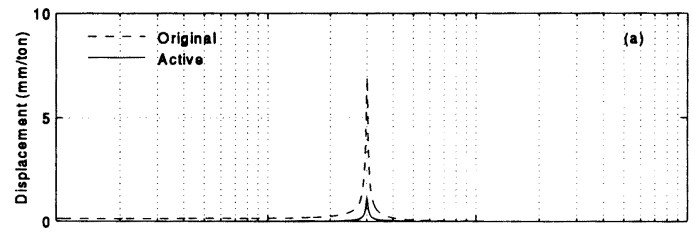

(a ) wind gust

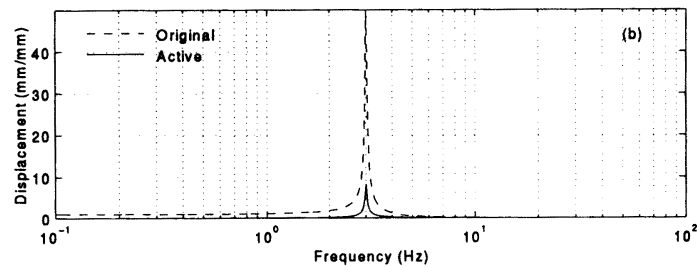

(b) support oscillation

Fig. 8 Transfer function of the displacement for changing $\tau$ to $0.1 \mathrm{sec}$ subject to ( $\mathrm{a}$ ) wind gust ( $\mathrm{b}$ ) support oscillation 


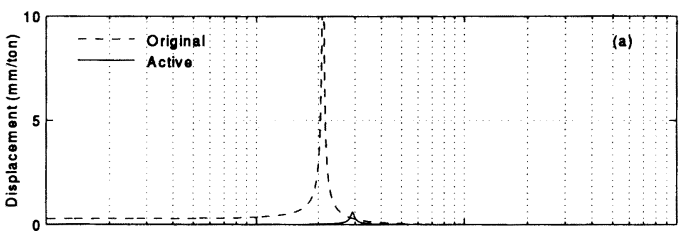

(a ) wind gust

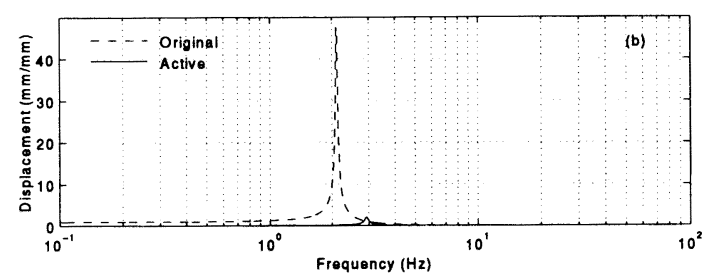

(b) support oscillation

Fig. 9 Transfer function of the displacement for changing $\omega_{n}$ to $2.1 \mathrm{~Hz}$ subject to ( a ) wind gust ( b ) support oscillation

\section{Conclusions}

A robust control design for the active vibration attenuation of the superstructure of ship using $\mathrm{H}_{\infty}$ control synthesis has been investigated. The excitations of the wind gust and hull vibration are considered in the numerical analysis to understand the performance of the control law design under this scheme. The results of the frequency and time domain analysis show that the effect of support oscillation is more severe than the wind gust for generating the superstructure vibration. However, the vibration induced by both excitations may be attenuated very effectively by the active control system with the designed controller. In addition, the performance of the active control system is analyzed under the conditions of system variations, including estimated error in the natural frequency of the structure and variation in time constant of the actuator. The results show that this designed system has maintains nearly the same efficiency even under large variations of the system parameters and the controller has superior performance in term of robust characteristic.

\section{Acknowledgment}

This research was sponsored by the National Science Council of the Republic of China under the grant NSC 86-2611-E-002-035.

\section{References}

1) Payer, H. G., and Asmussen, I., "Vibration Response on Propulsion-Efficient Container Vessels," Trans. SNAME, Vol. 93, 1985.

2) Kagawa, K., Yanagi, K., Shibata, M., Matsuo, M., and Yoshida, T., "Study on Active Vibration
Control for Ship and Engine Structure," Journal of the Society of Naval Architects of West Japan, No. 80, 1990.

3) Powell, M. J., Long, G., and Farrell, A., "Reduction of Hull Vibration in a Landing Craft by Means of a Vibration Absorber," RINA, 1980.

4) Matsuura, Y., Matsumoto, K., Mizuuchi, M., Arima, K., and Jouichi, H., "A study of Hydrodynamic Dampers to Reduce Ship Vibration," Journal of the Kansai Society of Naval Architects, Japan, Vol. 199, 1985.

5) Kulik, M., "Absorber with Auxiliary Force (AAF)," Ship Technology Research, Vol. 37, No. 2, 1990.

6) Hsueh, W. J., and Lee, Y. J., "A Hydraulic Absorber for Wideband Vibration Reduction in Ship Hulls," Journal of Offshore Mechanics and Arctic Engineering, ASME, Vol. 116, No. 1, 1994.

7) Kagawa, K., Koukawa, H., Fujita, K., Zensho, Y., and Matsho M., "Development of Tuned Liquid Damper for Ship Vibration," Journal of the Society of Naval Architects of West Japan, No.78, 1989.

8) Yoshida, Y., Yamauchi, S., Tanida, K., and Maeda, M., "Development of the Centrifugal Pendulum Absorber for the Reduction of Ship Superstructure Vibration," Journal of the Kansai Society of Naval Architects, Japan, Vol. 202, 1986.

9) Kondo, K., Ohta, T., and Satoh, H., "On the Vibration Control of Superstructures of Ships by Means of a Dynamic Vibration Absorber with an Adjustable Mass," Journal of the Society of Naval Architects of Japan, Vol. 162, 1987.

10) Ohtaka, K., Yamasaki, Y., and Yasuzawa, Y., "On the Active Control of Hull Vibration," Jour nal of the Society of Naval Architects of West Japan, No. 82, 1991.

11) Kakinouchi, T., Asano, T., Tanida, K., and Takahashi, N., "Active Mass Damper Demonstration for Ship Vibration Reduction," Naval Engineers Journal, 1992.

12) Takeda, Y., Kusumoto, H., Sasajima, H., Tanida, K., Koike, Y., Takahashi, N., and Iwanami T., "Development of Active Mass Damper for Ships by Hydraulic Control," Journal of the Society of Naval Architects of Japan, Vol. 171, 1992.

13) Safonov, M. G., Chiang R. Y., and Flashner, H., " $\mathrm{H}^{\infty}$ control Synthesis for a Large Space Structure," Proceedings of the American Control Conference, Atlanta, GA, 1988.

14) Yamato, H., Koyama, T., Fujita N., and Enomoto, M., "Roll Control System for the Hydrofoil Catamaran Based on the $\mathrm{H}_{\infty}$ Methodology," Journal of the Society of Naval Architects of Japan, Vol. 172, 1992.

15) Nakamura M., Kajiwara, H., Hyakudome, T., and Koterayama, W., "Design and Model Experiments on Thruster Assisted Mooring System," Journal of the Society of Naval Architects of Japan, Vol. 180, 1996.

16) Abdel-Rohman M., and Leipholz, H. E., "General Approach to Active Structural Control”, Journal of the Engineering Mechanics, ASCE, Vol. 105, No. 6, 1979. 